\title{
TECNOLOGIA ASSISTIVA, PESSOAS COM DEFICIÊNCIAS E O MERCADO DE TRABALHO
}

\author{
Cláudio Joaquim dos Santos Braga \\ Universidade Estácio (UNESA) \\ https://orcid.org/0000-0002-4060-7435 \\ Data de submissão: 10/07/2020 \\ Data de aprovação: 23/09/2020
}

\section{RESUMO}

Em um mundo cada vez mais informatizado, buscamos conhecer como a tecnologia assistiva pode auxiliar pessoas com deficiências diversas, na inclusão laboral. Justificamos a relevância do trabalho pois segundo Censo de 2010 (IBGE, 2010), temos 45 milhões de brasileiros com algum tipo de deficiência $(23,9 \%)$ da população o que equivale a um número muito maior de habitantes de diversos países. A tecnologia está cada dia mais inserida no cotidiano das pessoas e em especial no mercado de trabalho cada vez mais competitivo. O objetivo é apresentar a tecnologia assistiva como recurso tecnológico e legislação que permite flexibilização e adaptações para que o deficiente possa ter sua cidadania na integralidade conhecendo seus direitos fundamentais. A tecnologia assistiva como recurso de apoio pode ser extremamente eficiente, pois permite certo grau de autonomia e em diversos casos, plena condição de proporcionar ao deficiente executar uma tarefa laboral com extrema competência o que sem essa tecnologia seria reduzida. Utilizaremos como metodologia a pesquisa bibliográfica e a experiência profissional do autor que contribui há décadas na inclusão de deficientes no mercado de trabalho, através de projetos que visam a qualificação profissional, fornecendo condições via tecnologia assistiva para minimizar as dificuldades e maximizar as capacidades e competências no seguimento laboral, como exemplo um mouse trackball (mouse que possui uma bola em tamanho grande que permite seu uso por várias partes do corpo, para rolagem na tela e botões grandes para o clique) em grande parte, pode facilitar que um profissional com dificuldades para usar os membros superiores, utilize o recurso do mouse e mesmo com pouca mobilidade, com a mão, braço, os pés ou parte do corpo que permita seu uso. Como resultado apresentamos Leis que asseguram a inclusão e possibilitam o acesso de deficientes no mercado de trabalho. Concluímos que os resultados a serem alcançados poderão auxiliar futuros profissionais no uso do computador através de tecnologia assistiva, dando oportunidade ao deficiente de concorrer a uma vaga de trabalho nesse mercado cada vez mais informatizado.

Palavras-chave: Tecnologia assistiva. Pessoas com deficiências. Mercado de trabalho.

\section{ASSISTIVE TECHNOLOGY, PEOPLE WITH DISABILITIES AND THE LABOR MARKET}

\section{ABSTRACT}

In an increasingly computerized world, we seek to learn how assistive technology can assist people with diverse disabilities in job inclusion. We justify the relevance of the work 
because, according to the 2010 Census (IBGE, 2010), we have 45 million Brazilians with some type of disability (23.9\%) of the population, which is equivalent to a much larger number of inhabitants from different countries. Technology is increasingly inserted in people's daily lives and especially in the increasingly competitive job market. The objective is to present assistive technology as a technological resource and legislation that allows flexibility and adaptations so that the disabled can have their citizenship in full knowing their fundamental rights. Assistive technology as a support resource can be extremely efficient, as it allows for a certain degree of autonomy and, in several cases, is fully capable of enabling the disabled to perform a work task with extreme competence, which without this technology would be reduced. We will use as a methodology the bibliographic research and the professional experience of the author who has contributed for decades to the inclusion of disabled people in the labor market, through projects aimed at professional qualification, providing conditions via assistive technology to minimize difficulties and maximize capacities and competences in the field. labor follow-up, such as a trackball mouse (mouse that has a large size ball that allows it to be used by various parts of the body, for scrolling on the screen and large buttons for clicking) can, in large part, make it easier for a professional with difficulties to use the upper limbs, use the mouse feature and even with little mobility, with the hand, arm, feet or body part that allows its use. As a result, we present Laws that ensure inclusion and enable access for the disabled in the labor market. We conclude that the results to be achieved may help future professionals in the use of the computer through assistive technology, giving the disabled the opportunity to compete for a job vacancy in this increasingly computerized market.

Keywords: assistive technology, people with disabilities, labor market

\section{TECNOLOGÍA ASISTENCIAL, PERSONAS CON DISCAPACIDAD Y MERCADO LABORAL}

\section{RESUMEN}

En un mundo cada vez más informatizado, buscamos conocer cómo la tecnología asistencial puede ayudar a las personas con diversas discapacidades en la inclusión laboral. Justificamos la relevancia del trabajo porque, según el Censo de 2010 (IBGE, 2010), tenemos 45 millones de brasileños con algún tipo de discapacidad $(23,9 \%)$ de la población, lo que equivale a un número mucho mayor de habitantes de diferentes países. La tecnología se inserta cada vez más en la vida diaria de las personas y especialmente en el mercado laboral cada vez más competitivo. El objetivo es presentar la tecnología asistencial como recurso tecnológico y legislación que permita flexibilidad y adaptaciones para que las personas con discapacidad puedan tener su ciudadanía integral, conociendo sus derechos fundamentales. La tecnología asistencial como recurso de apoyo puede ser sumamente eficiente, ya que permite cierto grado de autonomía y, en varios casos, condiciones totales para que las personas con discapacidades puedan realizar una tarea laboral con extrema competencia, o que sin esta tecnología se vería reducida. Utilizaremos como metodología la investigación bibliográfica y la experiencia profesional del autor que ha contribuido durante décadas a la inclusión de las personas con discapacidad en el mercado laboral, a través de proyectos orientados a la calificación profesional, brindando condiciones a través de tecnologías asistivas para minimizar las dificultades y maximizar las capacidades y competencias en el campo laboral, como ejemplo un mouse trackball (ratón que posee una bola de gran tamaño que permite que sea utilizado por varias partes del cuerpo, para desplazarse por la pantalla y botones 
grandes para hacer clic) puede, en gran parte, facilitar a un profesional con dificultades la utilice las extremidades superiores, utilice la función del ratón e incluso con poca movilidad, con la mano, brazo, pies o parte del cuerpo que permita su uso. Como resultado, presentamos Leyes que garantizan la inclusión y permiten el acceso de las personas con discapacidad al mercado laboral. Concluimos que los resultados a alcanzar pueden ayudar a los futuros profesionales en el uso de la computadora a través de la tecnología asistencial, brindando a las personas con discapacidad la oportunidad de competir por una vacante laboral en este mercado cada vez más computarizado.

Palabras clave: Tecnología asistencial. Personas con discapacidades. Mercado laboral.

\section{INTRODUÇÃO}

No Brasil utilizamos o termo tecnologia assistiva, mas também podemos escutar e ler tecnologia de apoio ou ajuda técnica, principalmente em Portugal ou em países de língua espanhola. Esses termos são maneiras distintas de escrever a tecnologia assistiva. Por esse motivo utilizaremos em nosso Artigo o termo Tecnologia Assistiva (TA).

Importante afirmar que tecnologia não é algo recente, não necessariamente é algo do século XXI, ou está ligada à Internet ou a computadores. Lembramos o uso do fogo (que pode ser considerado uma tecnologia) que mudou o modo dos nossos antepassados e consequentemente foi um dos maiores trunfos da humanidade, pois ao dominar essa tecnologia, foi possível afastar predadores, aquecer-se nos períodos de frio, iluminar lugares escuros, utilizar na alimentação e em diversos outros usos.

O que dizer então de uma tecnologia de simples pedra talhada para ser uma ferramenta? Nossos ancestrais construíram diversas tecnologias que ajudaram a sobrevivência utilizando pedras e com o fogo os fizeram destacar em definitivo de outros animais e com a evolução chegamos até os dias atuais, criando cada vez mais tecnologias para o nosso cotidiano.

Poderíamos citar também grandes tecnologias que alavancaram nossa evolução como a roda e a escrita. Portanto podemos afirmar que o conceito tecnologia não está somente ligado a algo moderno e interligado aos computadores, ao digital e a novas tecnologias recentemente inventadas.

Resumidamente podemos dizer que tecnologia são instrumentos, métodos e técnicas que tem como objetivo a resolução de problemas diversos, melhorar nossa vida nos mais variados aspectos e que podem nos ajudar em nossas necessidades diárias (carros adaptados para deficientes, Língua Brasileira de Sinais (LIBRAS), leitura e escrita de textos em braille para cegos, métodos utilizados para ensino e aprendizado em determinadas disciplinas, roupas adaptadas, próteses, órteses, técnicas para utilizar adequadamente ferramentas adaptadas).

No Brasil, diversos textos versam sobre tecnologias assistivas para pessoas com deficiências, onde apresentamos algumas dessas Leis. Nossa Constituição apresenta diversos Artigos que versam sobre o tema conforme texto resumido na Cartilha da Procuradoria Federal dos Direitos do Cidadão, Brasília, setembro de 2004.

A nossa Constituição Federal elegeu como fundamentos da República a cidadania e a dignidade da pessoa humana (art. 10, inc. II e III), e como um dos seus objetivos fundamentais a promoção do bem de todos, sem preconceitos de origem, raça, sexo, cor, idade e quaisquer outras formas de discriminação (art. $3^{\circ}$, inc. IV). 
Garante ainda expressamente 0 direito à igualdade (art. $5^{\circ}$ ), e trata, nos artigos 205 e seguintes, do direito de TODOS à educação. Esse direito deve visar o pleno desenvolvimento da pessoa, seu preparo para o exercício da cidadania e sua qualificação para o trabalho (art. 205).

Além disso, elege como um dos princípios para o ensino, a igualdade de condições de acesso e permanência na escola (art. 206, inc. I), acrescentando que o dever do Estado com a educação será efetivado mediante a garantia de acesso aos níveis mais elevados do ensino, da pesquisa e da criação artística, segundo a capacidade de cada um (art. 208, V).

Portanto, a Constituição garante a todos o direito à educação e ao acesso à escola. Toda escola, assim reconhecida pelos órgãos oficiais como tal, deve atender aos princípios constitucionais, não podendo excluir nenhuma pessoa em razão de sua origem, raça, sexo, cor, idade, deficiência ou ausência dela." (BRASIL, 2004 p. 6).

Além das Leis, é importante destacar que vivemos em um século em que temos contato frequentes com tecnologias digitais diversas, em várias áreas do conhecimento e de nosso cotidiano.

Essa tecnologia disponível pode melhorar muito a qualidade de vida das pessoas deficientes, que conseguem ter acesso a uma tecnologia assistiva digital e usá-la adequadamente.

Lembramos que somente a tecnologia digital, o uso de computador ou Internet sem o uso de uma TA adequada pode construir um muro de impedimentos ao seu pleno uso pois a maioria desses equipamentos não foram desenvolvidos para manter uma Inclusão digital para os deficientes, embora muitos aparelhos e softwares já tenham em sua composição padrão, alguns módulos que facilitam e permitem seu uso para alguns tipos de deficiências. Alguns exemplos são leitores de tela, reconhecimento de voz em vários celulares.

Mesmo assim em casos em que o equipamento computadorizado ou digital não atenda de fábrica a utilização facilitada para deficientes, em muitos casos podemos nos valer de hardwares ou softwares que agregamos como substitutos de equipamentos tradicionais como a troca de um mouse comum (pequeno e que necessita de uma boa coordenação motora fina) por um mouse trackball (que permite o uso com parte dos membros superiores ou até com os membros inferiores) ilustrado na Figura 1.

Figura 1 - Mouse trackball

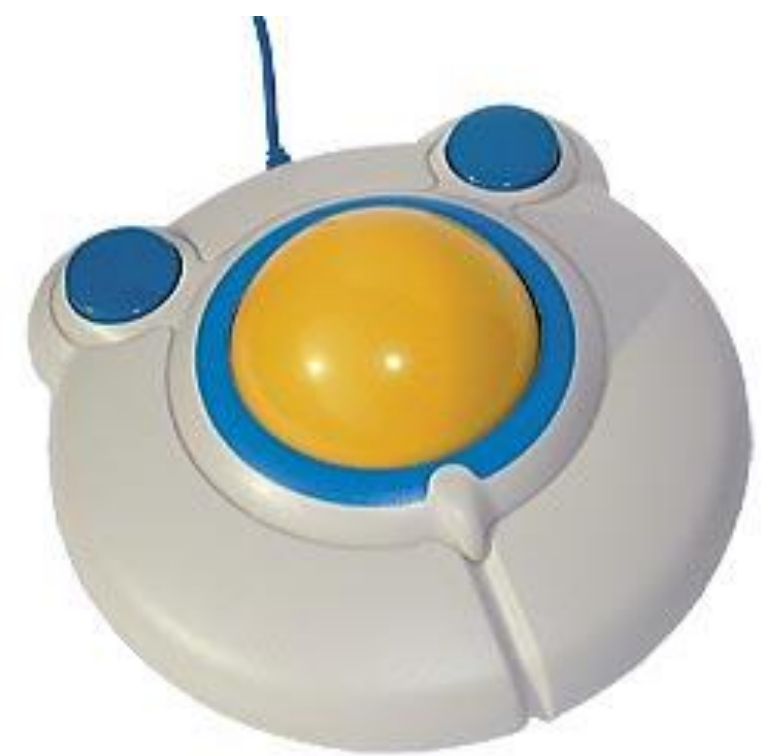

Fonte: https://images.dlf.org.uk/mee/products/full/0045488.jpg (2020). 
Com isso amplia-se a capacidade e habilidade do deficiente em seu uso e em alguns casos possibilita o uso do equipamento em igualdades de condições com uma pessoa que não possui limitações por conta de uma deficiência.

Em um mercado de trabalho competitivo e cada dia mais dependente da tecnologia é fundamental que todos, o que incluem pessoas com deficiências, estarem incluídos na alfabetização digital para o laboro. o que esbarra em diversos motivos físicos, metodológicos e oportunidades para vencer as limitações.

Entretanto, $\mathrm{o}$ acesso das pessoas com deficiência à alfabetização digital exige a remoção de barreiras físicas, metodológicas e financeiras. Embora a tecnologia ofereça diversos subsídios para vencer limitações impostas pela sociedade, muitos desses são desconhecidos ou não utilizados por falta de recursos para qualificar ou receber o deficiente no ambiente de trabalho adaptado.

No século XXI o difícil não está em como adquirir informação, mas sim como trabalhar a informação de maneira crítica. Freire sempre criticou a pedagogia bancária (FREIRE, 2014) e hoje em dia o professor já não é mais o dono das informações e saberes.

Perguntas podem ser respondidas instantaneamente pelo buscador Google. Esse não é o desafio, porém algo simples pode tornar-se difícil devido a limitações impostas pela sociedade que muitas pessoas com deficiências enfrentam diariamente.

Grande parte dos sistemas operacionais e sistemas utilizados em escritórios possuem recursos que auxiliam muitas pessoas em diversos tipos de deficiências. Citamos como exemplo o Sistema Operacional Microsoft que possui alguns comandos como:

- Lupa: que possui uma lupa virtual ampliando a área de visão com base no movimento do mouse.

- Narrador: que ativa um leitor de tela para que pessoas surdas ou com dislexia.

Em sistemas para escritórios considerados Office, muitos possuem corretores ortográfico que facilitam para dislexos e quem tem dificuldades motoras, alguns possuem sistemas de escrita ativados via voz ou de leitura para surdos, baixa visão ou dislexos. Acreditamos que temos que conhecer essas ferramentas e oferecer ao aprendiz esses recursos com a finalidade de dar autonomia e independência.

Sabemos que existem um número incontável de pessoas com deficiências; algumas congênitas e outras desenvolvidas ao longo da vida. Devemos conhecer as habilidades e capacidades em cada um desse indivíduos para que com eles possamos escolher uma tecnologia (assistiva) que permita o acesso e uso da tecnologia de forma adequada.

Grande parte das pessoas já convivem diretamente com a tecnologia incorporada com ou sem o nosso conhecimento. Hoje temos celulares que são tão potentes quanto muitos computadores e que podem ser utilizados como tecnologia assistiva com base no software escolhido.

Temos ainda televisores inteligentes, recursos embarcados em diversos sistemas que usamos no dia-a-dia e em muitas vezes nem percebemos que pode ser uma tecnologia assistiva como um simples controle remoto que pode ser utilizado para diversos equipamentos ou a tecla SAP usada em uma TV para que os surdos possam entender o que é exibido em sua tela, ilustrada na Figura 2. 
Figura 2 - Recurso da TV utilizando a tecla SAP

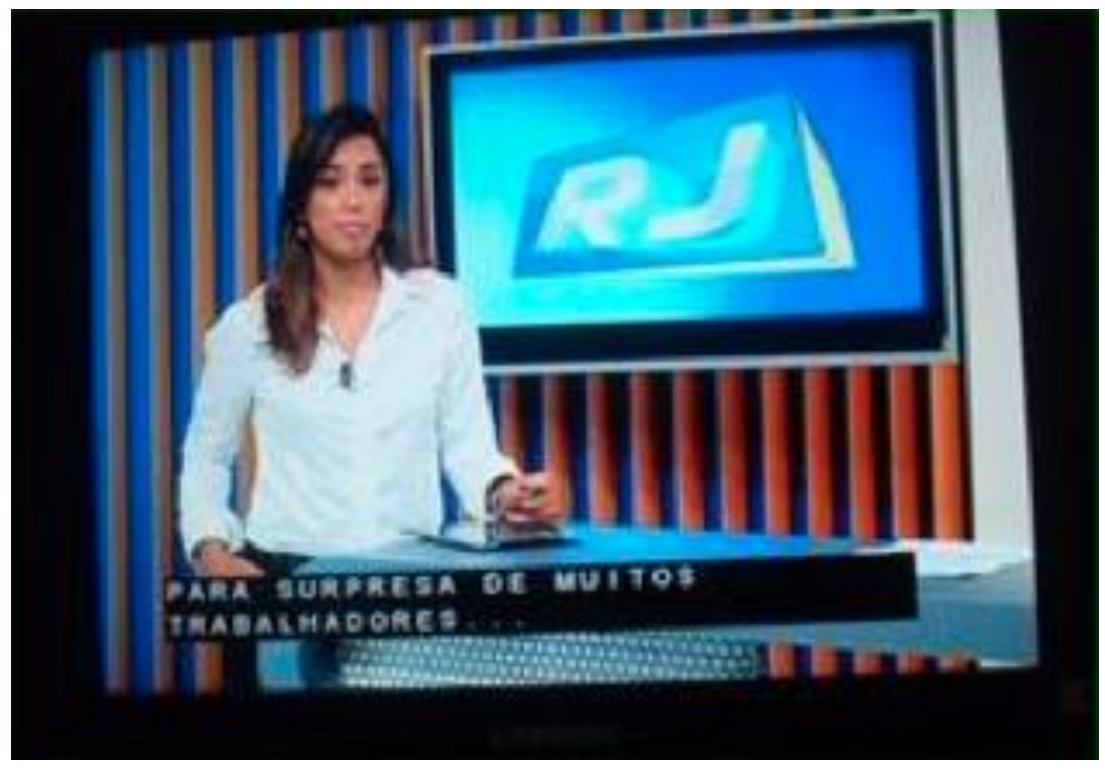

Fonte:

https://s2.glbimg.com/h0wuPR8BSBDPaFoSDfBzRbWDvBc=/s.glbimg.com/og/rg/f/original/2015/03/07/captu ra_de_tela_2015-03-07_as_13.41.19.png (2020).

A educação em todas as suas modalidades deve preparar todas as pessoas para a vida em sociedade o que inclui a possibilidade de ingressar no setor laboral.

O objetivo é apresentar a tecnologia assistiva como recurso tecnológico e legislação que permite flexibilização e adaptações para que o deficiente possa ter sua cidadania na integralidade conhecendo seus direitos fundamentais.

\section{FUNDAMENTAÇÃO TEÓRICA}

\subsection{Tecnologia Assistiva}

Ao buscarmos o termo Assistiva, em geral não encontramos em alguns dicionários físicos ou os que estão disponíveis online. Até nos processadores de textos em geral ao digitar essa palavra, ela aparece grifada de vermelho indicando que seu dicionário interno não conhece essa palavra. O mais próximo que encontramos quando pesquisamos assistiva é a palavra assistir, ajudar, acompanhar, o que não fica longe do sentido que imaginamos para esse nome assistivo, pois realmente nos faz alusão a ajudar, acompanhar, assistir no sentido de assistência.

Podemos citar que um simples pedaço de pau utilizado como uma bengala é uma tecnologia assistiva, com isso podemos mentalmente viajar pelo tempo e perceber muita tecnologia assistiva no decorrer de nossa história. Até uma história ou música que escutamos desde criança como o pirata da perna de pau, essa perna de pau também é uma tecnologia assistiva, pois permite ao pirata ficar de pé e andar.

Vemos com isso que o início de seu uso não é algo dos tempos modernos e sim vem desde os primórdios da humanidade onde o homem começou a utilizar objetos que 0 auxiliassem, assistissem, ajudassem a terem melhores condições e um nível de autonomia para realizar suas tarefas diárias. $\mathrm{O}$ que vêm de encontro com o conceito atual de tecnologia assistiva que em geral enfatiza levar certo nível de autonomia e independência ao deficiente físico. 
Interessante observar que essa expressão veio de uma tradução de um texto publicado em 1988 conforme citado por Bersch (2005, p.129 apud GALVÃO FILHO, 2009).

O termo Assistive Technology, traduzido no Brasil como Tecnologia Assistiva, foi criado oficialmente em 1988 como importante elemento jurídico dentro da legislação norte-americana, conhecida como Public Law 100-407, que compõe, com outras leis, o ADA - American with Disabilities Act. Este conjunto de leis regula os direitos dos cidadãos com deficiência nos EUA, além de prover a base legal dos fundos públicos para compra dos recursos que estes necessitam. Houve a necessidade de regulamentação legal deste tipo de tecnologia, a TA, e, a partir desta definição e do suporte legal, a população norte-americana, de pessoas com deficiência, passa a ter garantido pelo seu governo o benefício de serviços especializados e 0 acesso a todo o arsenal de recursos que necessitam e que venham favorecer uma vida mais independente, produtiva e incluída no contexto social geral.

Concordamos com Jatobá et al. (2018, p. 2): “A acessibilidade permite que as pessoas com deficiência utilizem os recursos com certa autonomia e, portanto, possibilita que a colaboração entre os usuários, quaisquer que sejam eles, seja favorecida".

\subsection{Legislação}

A lei No 10.098, de 19 de dezembro de 200 estabelece normas gerais e critérios básicos para a promoção da acessibilidade das pessoas portadoras de deficiência ou com mobilidade reduzida, e dá outras providências.

Art. 10 Esta Lei estabelece normas gerais e critérios básicos para a promoção da acessibilidade das pessoas portadoras de deficiência ou com mobilidade reduzida, mediante a supressão de barreiras e de obstáculos nas vias e espaços públicos, no mobiliário urbano, na construção e reforma de edifícios e nos meios de transporte e de comunicação.

Decreto № 3.956, de 08 de outubro de 2001 promulga a Convenção Interamericana para a Eliminação de Todas as Formas de Discriminação contra as Pessoas Portadoras de Deficiência. Esse Decreto é muito importante, pois define o que é deficiência e o objetivo a ser alcançado:

\section{Deficiência}

O termo "deficiência" significa uma restrição física, mental ou sensorial, de natureza permanente ou transitória, que limita a capacidade de exercer uma ou mais atividades essenciais da vida diária, causada ou agravada pelo ambiente econômico e social.

\section{$[\ldots]$}

Artigo II

Esta Convenção tem por objetivo prevenir e eliminar todas as formas de discriminação contra as pessoas portadoras de deficiência e propiciar a sua plena integração à sociedade.

Decreto № 6.949, de 25 de agosto de 2009 promulga a Convenção Internacional sobre os Direitos das Pessoas com Deficiência e seu Protocolo Facultativo, assinados em Nova York, em 30 de março de 2007. Destaco os seus princípios gerais:

\section{Artigo 3}

Princípios gerais

Os princípios da presente Convenção são:

a) $O$ respeito pela dignidade inerente, a autonomia individual, inclusive a liberdade de fazer as próprias escolhas, e a independência das pessoas;

b) A não-discriminação;

c) A plena e efetiva participação e inclusão na sociedade; 
d) O respeito pela diferença e pela aceitação das pessoas com deficiência como parte da diversidade humana e da humanidade;

e) A igualdade de oportunidades;

f) A acessibilidade;

g) A igualdade entre o homem e a mulher;

h) O respeito pelo desenvolvimento das capacidades das crianças com deficiência e pelo direito das crianças com deficiência de preservar sua identidade.

Apresentamos um marco importante aconteceu no dia 16 de novembro de 2006, quando a Secretaria Especial dos Direitos Humanos da Presidência (SEDH/PR) o Secretário Especial dos Direitos Humanos da Presidência da República, o Sr. Paulo de Tarso Vannuchi, publicou a portaria no 142, a qual institui o Comitê de Ajudas Técnicas (CAT).

Com base nessas responsabilidades em reunião nos dias 13 e 14 de dezembro de 2007 no Hotel Nacional em Brasília - DF, foi realizado um encontro cujo objetivo foi discutir a Tecnologia Assistiva voltada às pessoas com deficiências. Segundo a Ata VII Reunião do Comitê de Ajudas Técnicas (CAT) com Coordenadoria Nacional para Integração da Pessoa Portadora de Deficiência (CORDE), Secretaria Especial dos Direitos Humanos (SEDH) e a Presidência da República apresenta e o texto que hoje define o conceito de Tecnologia Assistiva aqui no Brasil:

Tecnologia Assistiva é uma área do conhecimento, de característica interdisciplinar, que engloba produtos, recursos, metodologias, estratégias, práticas e serviços que objetivam promover a funcionalidade, relacionada à atividade e participação, de pessoas com deficiência, incapacidades ou mobilidade reduzida, visando sua autonomia, independência, qualidade de vida e inclusão social. (BRASIL - SDHPR. - Comitê de Ajudas Técnicas - ATA VII - 2007, p. 3)

Desmembrando um pouco essa definição "é uma área de conhecimento interdisciplinar": Impossível pensar em tecnologia assistiva sem considerar os diversos profissionais que podem e devem estar envolvidos em sua atividade. Exemplo: precisamos de professores, fisioterapeutas, engenheiros, arquitetos, técnicos de informática, fonoaudiólogos, psicopedagogos, neurocientistas, ortopedistas, etc.

Por isso essa forma interdisciplinar é muito importante e dependendo da necessidade ou capacidade da pessoa com deficiência precisamos interagir com diferentes disciplinas, profissões e profissionais "que engloba produtos, recursos, metodologias, estratégias, práticas e serviços" para encontrarmos uma solução - Como podemos imaginar são inúmeros produtos desenvolvidos e em geral para cada deficiência, encontramos um tipo de produto específico, importante saber que o mesmo produto para a mesma deficiência em muitas vezes por conta de características individuais ainda necessitamos fazer ajustes para melhor atender o bem estar da pessoa.

Da mesma forma os recursos, métodos, estratégias, práticas e serviços devem ser direcionados e sempre que possíveis individualizadas para melhor adaptar ao deficiente, mas com ênfase em integrar no convívio social com os não deficientes. "que objetivam promover a funcionalidade, relacionada à atividade e participação, de pessoas com deficiência, incapacidades ou mobilidade reduzida, visando sua autonomia, independência, qualidade de vida e inclusão social".

Resumidamente a intenção da tecnologia assistiva é dar autonomia, independência e qualidade de vida e inclusão social, dando condições para que o deficiente possa conviver socialmente em sua comunidade de maneira autônoma, conseguindo realizar as atividades cotidianas com maior independência, possibilitando uma melhor integração social na comunidade em geral.

Decreto № 7.612, de 17 de novembro de 2011 - Institui o Plano Nacional dos Direitos da Pessoa com Deficiência - Plano Viver sem Limite. Apesar de existirem 
diversas Leis em nosso país ainda existe um abismo que distancia pessoas com e sem deficiência e entre esse abismo encontramos também diversos recursos ligados à tecnologia e ao mercado de trabalho.

É inegável o avanço e o uso das tecnologias nos dias atuais, porém levamos em considerações que o Brasil apresenta diferentes realidades o que nos leva a termos muitos profissionais afastados da tecnologia e mais distantes de muitos empregos disponíveis no mercado de trabalho.

Esses profissionais necessários ao mercado de trabalho deveriam começar a sua formação nos bancos escolares, porém com base no relatório do Censo Escolar (INEP, 2016), ainda vivenciamos problemas encontrados em séculos anteriores.

Segundo Padilha e Abranches (2010 apud SOARES; ALMEIDA, 2020, p. 85-86) "ao refletir sobre educação em direitos humanos e novas tecnologias, se observa a realidade do aparelhamento tecnológico precário das escolas, pois ainda que haja investimento para aparelhar as escolas, com computadores e tablets."

Corroborando com o contexto, a pesquisa de Paiva (2010) "apontou para a carência estrutural de várias escolas em Minas Gerais, que servem de espelho para que se observem as escolas brasileiras, sem infraestrutura mínima de funcionamento para o uso e manutenção das tecnologias informacionais"

No meio de tanta desigualdade reflete diretamente na qualidade da educação e diretamente na formação de muitos profissionais que sem uma qualificação desejada pelo mercado de trabalho fica em uma situação de ostracismo laboral. Se considerarmos que em todo esse cenário temos pessoas com deficiências físicas e intelectuais o problema se agrava exponencialmente.

\section{METODOLOGIA}

Utilizamos como metodologia a pesquisa bibliográfica e a experiência profissional do autor que contribui há décadas na inclusão de deficientes no mercado de trabalho com a aplicação de cursos para professores e profissionais de Recursos Humanos. Além de participar como professor lecionando classes sobre o tema da tecnologia assistiva, demonstrando diversos recursos digitais ou não para o uso do deficiente, que permite um melhor desempenho em sua autonomia e qualidade de vida, valorizando as capacidades e minimizando as limitações.

\section{CONSIDERAÇÕES FINAIS}

Com base na análise bibliográfica percebemos que existem diversas Leis que resguardam o direito das pessoas com deficiências e que em seus parágrafos legitimam uma intenção de igualdade oportunizando direitos para minimizar os efeitos de limitações físicas e intelectuais em um mundo não preparado na prática para esses cidadãos.

A tecnologia assistiva é uma alternativa que pode auxiliar em diversas possibilidades e permitir o ingresso de muitos deficientes em cargos antes destinados aos deficientes e não ocupados por uma não adaptação que permita uma autonomia laboral e também a cargos gerais em que o deficiente com 0 auxílio da TA pode normalmente pleitear e conquistar a vaga desejada.

Percebemos que em muitos casos os deficientes e os empregadores desconhecem seus recursos e conscientemente ou inconscientemente ignoram suas 
possibilidades, com isso seu potencial para diminuir o abismo entre as pessoas com e sem deficiência.

Notamos que apesar de ter leis que garantem vagas para deficientes, muitas não são utilizadas por falta de qualificação profissional e orientações para uso adequado de tecnologias assistivas no ambiente laboral.

Acreditamos que o uso de tecnologia assistiva para pessoas com deficiências é o diferencial que pode fazer com que ela possa trabalhar em condições de igualdade em alguns casos com os que não apresentam deficiências, permitindo disputar e obter vagas antes somente utilizadas por pessoas não deficientes.

Devemos aproveitar os recursos tecnológicos para unir, integrar, socializar e compartilhar conhecimentos, permitindo que o aluno seja usuário e autor de conteúdos e atividades, participando sempre de forma ativa apoderando-se dos recursos para um uso educativo dessas ferramentas tecnológicas.

Esse uso pleno além de fornecer uma autonomia possibilita que a pessoa se sinta mais integrada socialmente, pois a tecnologia permite aproximar pessoas outrora distantes para uma convivência além do mundo virtual.

O mercado de trabalho possui muitas vagas que poderiam ser preenchidas por deficientes, mas as vezes um simples teclado torna-se um limitador dessa conquista.

Vemos, portanto, a importância da tecnologia assistiva em todos os momentos da vida laboral de pessoas que são deficientes físicos e dependem de ajudas técnicas para exercerem uma profissão de maneira adequada com qualidade e competência.

\section{REFERÊNCIAS}

BERSCH, R. Introdução à Tecnologia Assistiva. In: SEMINÁRIO REGIONAL DE FORMAÇÃO DE GESTORES E EDUCADORES. EDUCAÇÃO INCLUSIVA: DIREITO À DIVERSIDADE, 2., 2005. Anais... Secretaria Municipal de Educação de Presidente Prudente. São Paulo: SEESP/MEC. 2005.

BRASIL. Constituição (1988). Constituição da República Federativa do Brasil. Brasília, DF: Senado Federal: Centro Gráfico, 1988.

BRASIL. DECRETO № 3.956, DE 8 DE OUTUBRO DE 2001. Promulga a Convenção Interamericana para a Eliminação de Todas as Formas de Discriminação contra as Pessoas Portadoras de Deficiência. Disponível em: <http://www.planalto.gov.br/ccivil_03/decreto/2001/d3956.htm>. Acesso em: 10 jul. 2020.

\section{BRASIL. DECRETO № 5.296 DE 2 DE DEZEMBRO DE 2004. Regulamenta as Leis} 10.048 , de 8 de novembro de 2000, que dá prioridade de atendimento às pessoas que especifica, e 10.098, de 19 de dezembro de 2000, que estabelece normas gerais e critérios básicos para a promoção da acessibilidade das pessoas portadoras de deficiência ou com mobilidade reduzida, e dá outras providências. Disponível em: <http://www.planalto.gov.br/ccivil_03/_ato2004-2006/2004/decreto/d5296.htm>. Acesso em: 10 jul. 2020.

BRASIL. DECRETO № 6.949, DE 25 DE AGOSTO DE 2009. Promulga a Convenção Internacional sobre os Direitos das Pessoas com Deficiência e seu Protocolo Facultativo, assinados em Nova York, em 30 de março de 2007. Disponível em: 
<http://www.planalto.gov.br/ccivil_03/_ato2007-2010/2009/decreto/d6949.htm>. Acesso em: 10 jul. 2020.

BRASIL. DECRETO № 7.612, DE 17 DE NOVEMBRO DE 2011. Institui o Plano Nacional dos Direitos da Pessoa com Deficiência - Plano Viver sem Limite. Disponível em: <http://www.planalto.gov.br/ccivil_03/_Ato20112014/2011/Decreto/D7612.htm>. Acesso em: 10 jul. 2020.

BRASIL. LEI № 10.098, DE 19 DE DEZEMBRO DE 2000. Estabelece normas gerais e critérios básicos para a promoção da acessibilidade das pessoas portadoras de deficiência ou com mobilidade reduzida, e dá outras providências. Disponível em: <http://www.planalto.gov.br/ccivil_03/Leis/L10098.htm>. Acesso em: 10 jul. 2020.

BRASIL. Ministério Público Federal. $\mathrm{O}$ acesso de alunos com deficiência às escolas e classes comuns da rede regular. 2004. Disponível em: <http://pfdc.pgr.mpf.mp.br/atuacao-e-conteudos-de-apoio/publicacoes/pessoa-comdeficiencia/acesso_alunos_ensino_publico_2004>. Acesso em: 10 jul. 2020.

FREIRE, P. Pedagogia da autonomia: Saberes necessários à prática educativa - Editora Paz e Terra, 2014.

GALVÃO FILHO, T. A. Tese de Mestrado. Tecnologia Assistiva para uma Escola Inclusiva: Apropriação, Demandas e Perspectivas. Salvador. Bahia. 2009. Disponível em: <https://www2.ufff.br/nai//files/2009/07/Tese-Teofilo-Galvao.pdf>. Acesso em: 10 jul. 2020.

IBGE. Instituto Brasileiro de Geografia e Estatística. Censo Demográfico - Censo 2010 Características gerais da população, religião e pessoas com deficiências. Principais resultados. Disponível em: https://www.ibge.gov.br/estatisticas/sociais/populacao/9662censo-demografico-2010.html?edicao=9749\&t=destaques Acesso em: 10 dez. 2020.

INEP. Instituto Nacional de Estudos e Pesquisas Educacionais Anísio Teixeira. Censo Escolar 2016. Disponível em: https://www.gov.br/inep/pt-br/acesso-a-informacao/dadosabertos/microdados/censo-escolar Acesso em: 10 dez. 2020.

JATOBÁ, A.; MARTINS B.; SILVA, M. L. da; DA SILVA, V. F. Relações entre acessibilidade e colaboração em três ambientes virtuais. Revista Carioca de Ciência, Tecnologia e Educação (online). Rio de Janeiro: v. 3, n. 2, 2018. Disponível em: $<$ https://recite.unicarioca.edu.br/rccte/index.php/rccte/article/view/52/79>. Acesso em: 10 jul. 2020.

PAIVA, L. L. de. Do giz colorido ao data show: uma conexão desconectada. Juiz de Fora: UFJF, 2010.

SOARES, M. de F.; ALMEIDA, V. E. de. A educação em direitos humanos e as novas tecnologias para o exercício da cidadania. Revista Carioca de Ciência, Tecnologia e Educação (online). Rio de Janeiro: v.5, n.1, 2020. E-ISSN 2596-058X. Disponível em: $<$ https://recite.unicarioca.edu.br/rccte/index.php/rccte/article/view/98/117>. Acesso em: 10 jul. 2020. 